\title{
Dielectric Elastomer Based “Grippers” for Soft Robotics
}

\section{Citation}

Shian, Samuel, Katia Bertoldi, and David R. Clarke. 2015. Dielectric Elastomer Based "Grippers" for Soft Robotics. Advanced Materials. Forthcoming.

\section{Published Version}

10.1002/adma.201503078

\section{Permanent link}

http://nrs.harvard.edu/urn-3:HUL.InstRepos:17694369

\section{Terms of Use}

This article was downloaded from Harvard University's DASH repository, and is made available under the terms and conditions applicable to Open Access Policy Articles, as set forth at http:// nrs.harvard.edu/urn-3:HUL.InstRepos:dash.current.terms-of-use\#OAP

\section{Share Your Story}

The Harvard community has made this article openly available.

Please share how this access benefits you. Submit a story.

Accessibility 


\title{
Dielectric Elastomer Based "Grippers" for Soft Robotics
}

Samuel Shian*, Katia Bertoldi, and David R. Clarke

\author{
S. Shian, K. Bertoldi and D. R. Clarke \\ John A Paulson School of Engineering and Applied Sciences \\ Harvard University \\ 11 Oxford St. \\ Cambridge, MA 02138, USA \\ E-mail: sshian@seas.harvard.edu
}

Keywords: grippers, soft robotics, dielectric elastomer actuators, bending, fiber stiffening

The actions of human hands and animal's feet provide the inspiration for many robotic actuators and devices. Of these actions, one of the most basic is gripping different objects. For instance, the use of fingers to grip a wide range of differently shaped objects, ranging from chop sticks to rugby balls. Similarly, bird's feet are used to hold onto objects, such as branches, and also to grasp prey. In the most general sense, these might all be classified as examples of "grippers". The simplest mechanical analog of a finger is the bending of a cantilever beam in which the displacement is a function of some control variable. Strictly speaking the dexterity of fingers comes about because each can be moved independently and because each is an articulated structure consisting of three beams in series rotating about the knuckles enabling a larger range of motion and adaptability to simple shapes. These actions are embodied in many standard industrial robots, for instance those used in car assembly and on other manufacturing lines, and are usually actuated using hydraulics and electric motors to produce the large range of forces and motions required. In translating the underlying concepts to devise robotics that interact directly with humans, attention has focused on soft robotics where the mechanical interface with the human body is soft, yielding and compliant, characteristics associated with elastomers. These mechanical attributes of soft materials have the potential for a more gentle interaction with fragile objects while also being tolerant to potentially damaging forces, such as impact. 
In addition to being made of soft, elastic materials it is also desirable that soft robots are capable of continuous deformation without the need of complex joints. This has led to the development of pneumatically actuated soft robotics ${ }^{[1]}$ in which air pressure not only provides continuous actuation as well as forces over large distances but provides local conformability by acting on a soft skin. Ideally, a soft robot also has the characteristics that the deformation of the contact surfaces can be preprogrammed into the body shape, thereby simplifying control mechanism for performing complex motions. ${ }^{[2]}$ A new approach to achieving these goals using dielectric elastomer actuators is described in this work and is based on incorporating a few stiff fibers to direct the actuation shape. The purpose of the fibers is to alter the shape functionality in response to a voltage rather than to alter the material properties, which is the usual reason for incorporating fibers into a material. ${ }^{[3,4]}$

In the last decade or so, there has been considerable interest in using dielectric elastomer actuators as artificial muscles for soft-robotics since dielectric elastomers have similar mechanical properties as human skin, notably a low elastic modulus and a large strain capability, and their actuation can be controlled by the application of an electrical voltage. ${ }^{[5-9]}$ The last simplifies the infrastructure necessary for their implementation, eliminating the need of air pumps or compressors and gas valves typically found in pneumatics actuators. Because of its simplicity in controls, dielectric elastomers could enable untethered operation of soft robots while maintaining the overall compliance of the structure.

In this work, we illustrate a number of "gripper" actions made possible by incorporating a few stiff fibers into voltage-actuated dielectric elastomer beams. It is found that only a few, in some cases a single fiber, can be used to break the symmetry of the electric field induced deformation as well as to pre-determine the direction of shape changes. Examples are shown 
in Figure 1 and by the accompanying videos in the supplementary materials. In the first example, a wrap-around gripper using vertically aligned fibers is shown to pick and place a cylindrical metallic object and a grape (Figure 1a). In the second example, a dielectric elastomer gripper having horizontally aligned fibers is shown to grip and lift a wood-beam structure (Figure 1b). The emphasis in this work on using a small number of fibers is significant since incorporating too high a concentration increases the stiffness and detracts from the soft mechanical response desirable of soft robotics. Previous approaches use prestretched dielectric elastomers which require relatively bulky and stiff frames to maintain structural integrity, causing increase in weight and more importantly reducing the overall flexibility or soft nature of the system. ${ }^{[10,11]}$

The basis of dielectric elastomer actuation is well established and can be summarized as follows. ${ }^{[12]}$ When a voltage is applied to a sheet of elastomer, with thin electrodes on either side, the elastomer becomes thinner in response to the Maxwell stress derived from the electrostatic attraction between the charges on the opposing electrodes. This attraction exists in all capacitors but because the mechanical stiffness of elastomers can be twelve to fifteen orders of magnitude smaller than that of conventional capacitor materials, typically oxides, the electric field induced displacements are commensurately larger. Furthermore, as elastomers are incompressible materials, their volume doesn't change upon actuation and so the contractions parallel to the applied field are compensated by stretching in the perpendicular directions. These changes in dimensions provide for a voltage-tunable mechanical actuation. The Maxwell stress is given by $\sigma_{z}=-\varepsilon E^{2}$, where $E$ is electrical field and $\varepsilon$ is the permittivity of the elastomer layer. 
An intrinsic feature of the parallel plate actuation is that the stresses, and consequently the stretches, produced are equal in both perpendicular directions, $\sigma_{x}=\sigma_{y}=-1 / 2 \sigma_{z}$, namely they are equi-biaxial. While this is an attractive attribute for some applications, for instance when used as a membrane pump or an adaptive lens, ${ }^{[13,14]}$ it is a limitation when linear actuation or translation in only one direction is desired. To overcome this, a number of strategies have been devised. One of these is to construct a cylindrical actuator consisting of an elastomer sleeve attached to a series of stiff rings. ${ }^{[15]}$ The rings constrain the expansion of the elastomer parallel to the rings and convert it to an increased stretch in the perpendicular direction, along the axis of the cylinder to produce an axial actuator. Equivalently, a stiff strip or fiber attached to the elastomer prevents the elastomer expanding along the strip and since the volume of the elastomer sheet cannot change with applied voltage, the elastomer stretches in a direction perpendicular to the strips instead ${ }^{[16]}$. In this work, we take advantage of this constraint, produced parallel to the fibers, to pre-determine the direction in which the actuator structure deflects. This forms the basis for the actuation actions shown in Figures 1-3.

One method of converting the voltage-induced lateral expansion to a flexural actuator is to bond the elastomer sheet, electroded on both sides, to a sheet of another, passive material. Figure 2a. When a voltage is applied across the elastomer sheet it expands laterally, applying a bending moment to the whole bilayer. Formally, this is a unimorph actuator, since the bending curvature can only be of one sign, and in the absence of any elastic anisotropy forms a concave surface. If the two layers are narrow, parallel strips, then the structure is, in effect, a long narrow beam and when a voltage is applied, the beam deflects perpendicular to the length of the beam. The deflection of the beam is plane-strain and can be analyzed using the Timoshenko analysis, introduced in 1925, to describe the deformation of bi-metallic strips used for over a hundred years as the basis of thermally-activated electrical circuit switches, 
for instance in thermostats ${ }^{[17]}$. However, actuation of this narrow beam configuration, although operationally similar to the "cocking" of a finger, is not a very practical geometry for voltage actuation because it is prone to electrical breakdown around the edges from one electrode to the other. Consequently, large voltage-induced deflections cannot be obtained before breakdown occurs. The practical solution to the problem of breakdown around the edges is to use a smaller electrode area so that the electrodes do not extend all the way to the edges of the beam. While this design approach can be effective in increasing the field until breakdown occurs, it leaves a "picture-frame" border of elastomer that is not actuated.

The other limiting geometry is when the two sheets are both large in extent compared to their thicknesses, for instance a square or rectangular shaped bi-layer. When a voltage is applied, the induced lateral expansion causes the originally flat bilayer to become concave. (In the special case of where the sheets are circular, the actuated shape is in the form of a hemispherical cap). ${ }^{[18,19]}$ The response to successively higher voltages is shown in the finite element simulations of a square sheet in Figure 2 for two cases, one where the electrodes extend all the way to the edges (Figure 2d) and the other where there is a border around the edges that is not electrode (Figure $2 \mathrm{e}-\mathrm{g}$ ). In both cases, the deformation exhibits the same four-fold symmetry as the square geometry of the sheets and with "dog-ear" deformation at the corners. Near the center, the two orthogonal curvatures are indistinguishable consistent with the formation of a hemispherical cap (Figure 2c). As can also be seen, the un-electroded borders decrease the out-of-plane displacements but do not alter the symmetry. The deformation increases with the applied voltage, $V$, and scales with the parameter, $E \sqrt{\varepsilon / \mu}$, where $E$ is the nominal electric field, $E=V / H, \varepsilon$ and $\mu$ are the dielectric constant and shear modulus, respectively. ${ }^{[12,20]}$ The parameter $E \sqrt{\varepsilon / \mu}$ is dimensionless and essentially represents the actuation strain. The border constraint is due to two different effects, one on 
bending and the other on stretching: as the border region does not thin whereas that of the electrode material decreases it's bending stiffness remains constant, and also having a larger cross-section it requires more energy to stretch.

Experiments and simulations show that by embedding a single stiff fiber placed along one of the symmetry lines and located at the interface between the active and passive layers the voltage-induced, four-fold displacement symmetry is broken to produce a two-fold bending symmetry. For large ratios of the fiber to elastomer stiffness, the symmetry line is always parallel to the direction of the fiber and the beam bends about the fiber, as shown in Figure $2 \mathrm{~g}$. This also occurs when the fiber is placed at the outer surface of the active layer rather than in the neutral plane or in general when the fiber is placed anywhere within the bilayer except on the symmetry line. However, simulations suggest that there is a minimum stiffness ratio below which the displacements are, instead, primarily perpendicular to the fiber direction, as shown in Figure 2f. This is illustrated by the behavior of the two orthogonal curvatures computed, in the vicinity of the center of the square, as a function of the electric field parameter for different ratios of the fiber to elastomer stiffness (Figure 2c). (In these simulations, the fibers have a square cross-section $\mathrm{H} / 10$ on side). For low stiffness ratios, up to 5,000 for these examples, the two curvatures are the same until they abruptly bifurcate at a critical field, as indicated by stiffness ratio of 50 in Figure 2c. With increasing stiffness ratio, similar bifurcation behavior occurs but the bifurcation occurs at progressively smaller values of the normalized electric field. So, except for the special case of the fiber being exactly along one of the symmetry lines, the bending always occurs about the fiber direction.

The foregoing analysis and results are for free-standing dielectric elastomer bilayers. However, when used in a device, the actuator has to be held, introducing additional constraints. For instance, in many applications, including when used as a cantilever beam, the 
actuator is clamped along one edge. This affects the deflections in the vicinity of the fixed end, becoming a progressively weaker influence further away. This is illustrated in the images in Figure 3 of the voltage induced deflection for four different bilayer beams, the first with no fiber, a second in which a few fibers are aligned at $90^{\circ}$, parallel to the clamped edge, a third at $60^{\circ}$ to the bottom edge of a rectangular beam, and at varying angle concentrically toward one of the lower corner. In the absence of any fiber, the bilayer beam deforms vertically, forming a vertical cylinder, Figure $3 \mathrm{a}$. This deformation is not very controllable since a slight misalignment of electrode placement or external forces could produce asymmetrical deformation and sometimes results in curls in preferentially from one corner. However, with a few aligned fibers, as small as one fiber, the beams do indeed deflect with a bending axis parallel to the fiber orientation as seen in the photographs of Figure 3. When the fibers are aligned perpendicular to the length of the beam, then, as shown in Figure $3 \mathrm{~d}$, as the deformation increases the beam can curl up and wrap around itself. This is the basis for the gripper in Figure 1b. For fiber oriented at $60^{\circ}$ to the bottom edge of a rectangular beam, the deformation begins at the corners well away from the clamped edge and the bending continues with increasing voltage until the clamped edge prevents further deformation.

The demonstration that one or more aligned fibers can determine the direction of the deflection of a beam containing a few fibers opens up a number of opportunities for the design of dielectric elastomer devices. Furthermore, examination of the images suggests that wider beams offer more shape conformability, more akin to webbed gloves than individual fingers. Two examples of this are the "pick and place" devices shown in Figure 1. As shown in the sequence of images at successively larger actuation voltages in Figure $3 \mathrm{~d}$, the beam not only bends perpendicular to the vertical fibers but the bottom edges curl inwards. This has the effect of the beam bending and conforming to the shape of the object, in this case both a cylindrical and a spherical object. 
The use of a few stiff fibers to determine the actuation geometry can be exploited in higher levels of design sophistication by using more than one array of aligned fibers. One example is using two sets of aligned fibers at right angles to one another but still within the plane of the bilayer. This is shown in Figure 1c, where the two layers are both electroded elastomers and with a set of fibers on the outer surface of each. Depending on which of the two elastomer layers is actuated with a voltage, the bending takes place perpendicular to the fiber orientation on the outer surface of the activated layer. Another example is a fan of fibers radiating away from a corner as in Figure 3g-h. In this arrangement, the bending curvature varies along the length of the fibers, forming a cone at high actuation strains.

The simulations and observations illustrate that it is probably more useful to consider the actuated deformations as being characterized by the continuous deformation of a series of tiled segments hinging and rotating about the individual fibers, as indicated by arrows in Figure $3 \mathrm{c}$ and $3 \mathrm{~d}$, rather than as a result of some increased anisotropic stiffness smeared throughout the active layer. The latter would result in the voltage-induced bending being a continuous function of position along the length of the beam. And, although, this is approached as the spacing between the fibers becomes similar to the unimorph thickness it doesn't adequately describe the local strains and deflections. It is more appropriate to consider that the fibers act as stiff structural ties constraining the deformation of a much softer material, much as the cytoskeletal filaments of actin fibers constrain the deformation of cells or how webbing is used to maintain the shape of fabrics and sails. ${ }^{[21]}$

In conclusion, we have shown that a variety of novel gripping actuators, suitable for soft robotics, can be devised by deliberately introducing one or more stiff fibers or strips in prearranged geometries so as to direct the bending direction of electrically actuated elastomer 
unimorphs. The function of the fibers is to not only to provide stiffening in specific directions but also act as geometric hinges in the shape changes. In essence, the fibers form a skeletal structure at an intermediate length scale that can be exploited in the design of more sophisticated shape change dielectric elastomer actuators. Finally, although the focus of this work has been on the shape changes themselves, these actuators could be used in conjunction with other soft robotics, such as pneumatic ones, where independent control or fine scale displacements may be beneficial.

\section{Experimental Section}

Materials and Experimental Procedures: The multilayer elastomer bending actuator was fabricated using a similar method as previously described. ${ }^{[22]}$ First, acrylic elastomers $(0.25$ $\mathrm{mm}$ thick, VHB 9473PC, 3M) were cut into $25 \times 50 \mathrm{~mm}$ sheets. In one sheet, carbon nanotubes electrodes were deposited on both of its surface using filtration-transfer method with Teflon filter. ${ }^{[23]}$ In this method, the electroded area $(22 \times 30 \mathrm{~mm})$ is defined using a template to leave uncoated margins of $1.5 \mathrm{~mm}$ along three sides and $18.5 \mathrm{~mm}$ along one side of the sheets. The long uncoated side is used as a fixed end of the beam and for creating electrical connection to external. The electroded sheet was bonded to another acrylic elastomer sheet to form the bilayer. Aluminum foil was used to connect the carbon nanotubes electrodes to the power supply wires. Vinyl fibers with width of $0.4 \mathrm{~mm}$ were cut from a $0.07 \mathrm{~mm}$ thick vinyl sheet using an automatic paper cutter (Cameo by Silhouette). These fibers were bonded using pressure on the electroded side of the bilayer. To minimize the tackiness of the VHB during gripping and releasing, a thin layer of silicone oil (AP 100, Dow Corning Corp.) was spread on gripping surface of the bilayer. The actuation voltage was provided by a high voltage power supply (Model 610E Trek, Medina, NY). 
To form the bimorph shown in Figure 1c, another layer of electrode and another layer of fibers were placed on the other side of the elastomer sheets so that either sheet can be independently actuated.

Finite Element Simulations: Numerical analyses were performed using the commercial nonlinear Finite Element package ABAQUS/Standard (version 6.12). Three dimensional models were built with dimension of $\mathrm{L} / \mathrm{H}=\mathrm{W} / \mathrm{H}=25 \mathrm{~mm} / \mathrm{mm}$ and meshed using linear brick elements (ABAQUS element type C3D8).The response of the dielectric material is captured by coding the free-energy function and its derivatives in a user-defined subroutine, UMAT. ${ }^{[24]}$ Moreover, we modelled the fibers as linear elastic. The Maxwell force is applied by defining a ground and a potential surface on opposite faces of the active elastomer layer. In this configuration, the electrodes are considered to have infinitesimal thickness and therefore to have negligible mechanical contribution to the bending actuator, which is a reasonable assumption for very thin SWNCT electrodes. The fibers with cross section of $0.1 \times 0.1 \mathrm{~mm}$ were structurally modeled using shell elements (element type S4R) and then merged with the elastomer using tie constraints. Mesh refinement study was performed to ensure accuracy.

\section{Acknowledgements}

This work was supported by the National Science Foundation through grant CMMI-1333835 and in part by the MRSEC program of the National Science Foundation under award number DMR 14-20570.

\section{References}

[1] F. Ilievski, A. D. Mazzeo, R. F. Shepherd, X. Chen, G. M. Whitesides, Angew. Chemie Int. Ed. 2011, 50, 1890.

[2] S. Kim, C. Laschi, B. Trimmer, Trends Biotechnol. 2013, 31, 287. 
[3] C. Bolzmacher, J. Biggs, M. Srinivasan, Proc. SPIE 2006, 6168, 616804.

[4] K. B. Subramani, E. Cakmak, R. J. Spontak, T. K. Ghosh, Adv. Mater. 2014, 26, 2949.

[5] R. Pelrine, R. D. Kornbluh, Q. Pei, S. Stanford, S. Oh, J. Eckerle, R. J. Full, M. A. Rosenthal, K. Meijer, in Smart Struct. Mater. 2002 Electroact. Polym. Actuators Devices, SPIE, 2002, pp. 126-137.

[6] C. Jordi, S. Michel, E. Fink, Bioinspir. Biomim. 2010, 5, 026007.

[7] S. Bauer, S. Bauer-Gogonea, I. Graz, M. Kaltenbrunner, C. Keplinger, R. Schwödiauer, Adv. Mater. 2013, 149.

[8] F. Carpi, D. De Rossi, R. Kornbluh, R. Pelrine, P. Sommer-Larsen, Dielectric Elastomers as Electromechanical Transducers: Fundamentals, Materials, Devices, Models and Applications of an Emerging Electroactive Polymer Technology, Elsevier, 2008.

[9] I. A. Anderson, T. Hale, T. Gisby, T. Inamura, T. McKay, B. O'Brien, S. Walbran, E. P. Calius, Appl. Phys. A Mater. Sci. Process. 2010, 98, 75.

[10] G. Kofod, W. Wirges, M. Paajanen, S. Bauer, Appl. Phys. Lett. 2007, 90, 081916.

[11] S. Araromi, I. Gavrilovich, J. Shintake, S. Rosset, H. Shea, SPIE Proc. 2014, 9056, 90562G.

[12] Z. Suo, Acta Mech. Solida Sin. 2010, 23, 549.

[13] S. Shian, R. M. Diebold, D. R. Clarke, Opt. Express 2013, 21, 8869.

[14] J. J. Loverich, I. Kanno, H. Kotera, Lab Chip 2006, 6, 1147.

[15] J. Huang, T. Lu, J. Zhu, D. R. Clarke, Z. Suo, Appl. Phys. Lett. 2012, 100, 211901.

[16] T. Lu, J. Huang, C. Jordi, G. Kovacs, R. Huang, D. R. Clarke, Z. Suo, Soft Matter 2012, 8, 6167.

[17] S. Timoshenko, J. Opt. Soc. Am. 1925, 11, 233.

[18] L. B. Freund, J. Mech. Phys. Solids 2000, 48, 1159.

[19] M. Finot, S. Suresh, J. Mech. Phys. Solids 1996, 44, 683.

[20] X. Zhao, Z. Suo, Appl. Phys. Lett. 2008, 93, 251902.

[21] R. Phillips, J. Kondev, J. Theriot, H. Garcia, Physical Biology of the Cell, Second Edition, Taylor \& Francis Group, 2012.

[22] S. Shian, K. Bertoldi, D. R. Clarke, SPIE Proc. 2015, 9430, 94301P. 
[23] S. Shian, R. M. Diebold, A. McNamara, D. R. Clarke, Appl. Phys. Lett. 2012, 101, 061101.
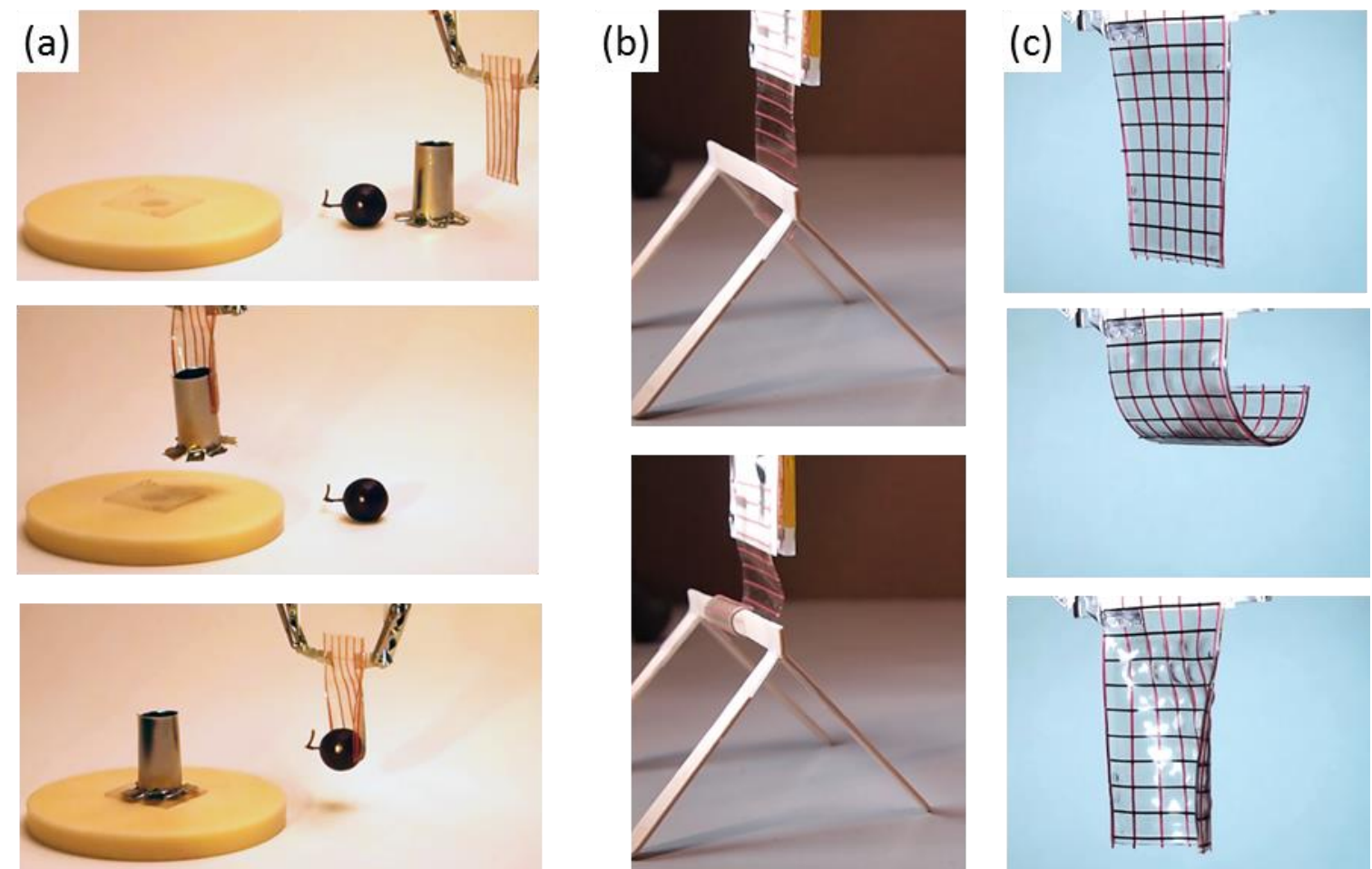

Figure 1. (a) Illustration of the action of a "pick-and-place" actuator made from an elastomer beam with a few vertical fibers. As can be more clearly seen in the accompanying videos, the elastomer bends to conform to the shape of both the cylinder and grape. (b) Another example of "pick-and-place" using horizontal wrap action. (c) A dual action actuator consisting of a bilayer of two dielectric actuators bonded together with fibers oriented in one direction in one layer and fibers oriented at 90 degrees in the other. When the side with horizontally aligned fiber is actuated, the bending curvature is vertical (second picture) whereas in third picture when the side with vertically aligned fiber is actuated, the bending curvature is horizontal. 

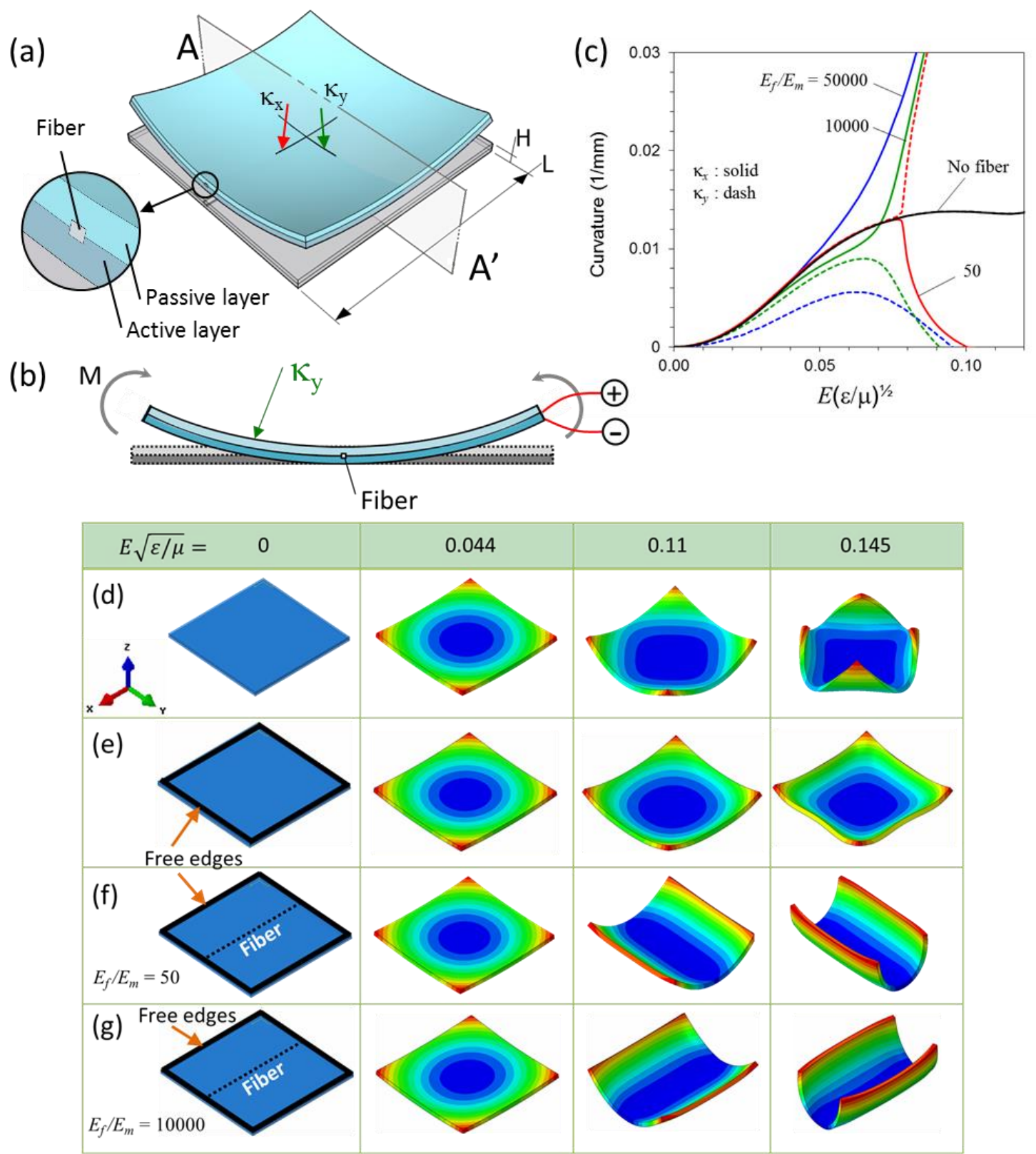

Figure 2. Finite element simulations of a square-shaped bilayer, consisting of a passive elastomer sheet bonded to a voltage actuated elastomer sheet $(\mathrm{L} / \mathrm{H}=25$, fiber width and heights are H/10). For comparison, the initial configuration of the bilayer is superimposed on the lower part shown in grey. The cross section A-A' is shown in (b) to indicate the location of fiber and the bending moment. The active layer is sandwiched between two compliant electrodes (not shown), which are connected to a voltage source. Note that the coverage of the compliant electrodes do not extent to the edge of the bilayer, instead there are narrow border (set to equal to $\mathrm{H}$ ) as free edges. (c) The two orthogonal curvatures of the bilayer, $\kappa_{\mathrm{x}}$ and $\kappa_{\mathrm{y}}$, are plotted as a function applied voltage at several fiber/elastomer stiffness ratio $\left(E_{f} / E_{m}\right)$. These curvatures are evaluated in the center region of the squares.

(d) In the absence of the fiber, the curvatures in the two-orthogonal directions are the same, which indicate that the shape deformation has the same four-fold symmetry of the square shape with largest deformation in the corners to produce the "dog-ear" distortion. (e) The symmetry is unaffected by a narrow border of the square that is not electrode although the displacements are reduced. (c and d) A single fiber on the neutral plane in the center of the square, breaks the deformation symmetry so that it has two-fold symmetry. For fiber/elastomer stiffness ratios up to 5,000 and shown for 50, the two curvatures, shown in full 
and dashed curves in (c), are the same until abruptly bifurcating. With increasing values of the stiffness ratio, the bifurcation takes place at progressively smaller fields. In each panel, the height displacement (z-axis) are color coded between red and blue denoting maximum and minimum values.
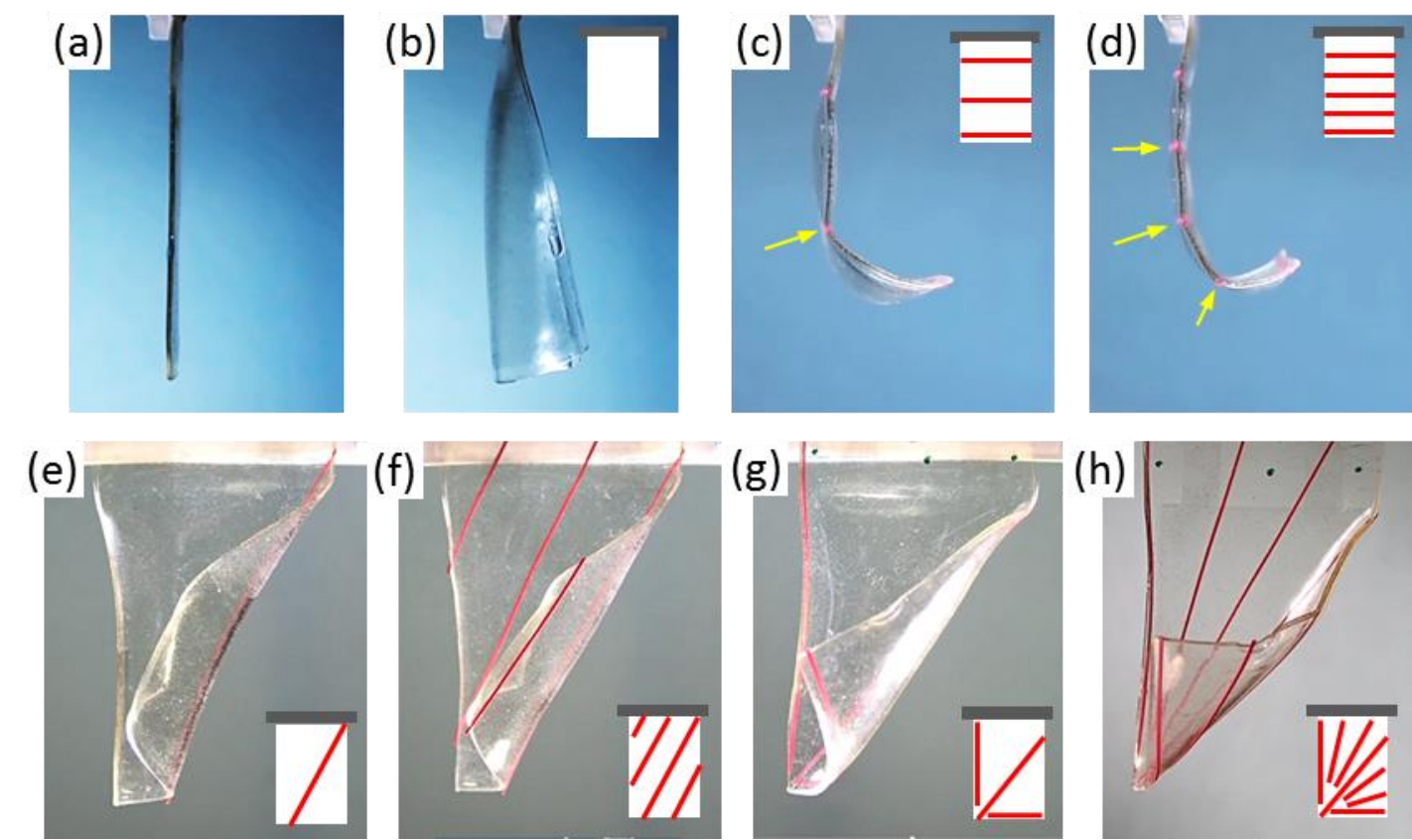

Figure 3. The shape of the bilayer under electrical potential for different fiber configurations, which are shown by the inset diagram. (a) The side view of the initial shape of the bilayer with no voltage. (b) In the absent of fiber, the bilayer bent in the horizontal direction, forming a vertical cylinder. (c) The addition of three horizontally aligned fiber changed the direction of the bending to be perpendicular to the fiber orientation. The fiber becomes inflection points, shown by the arrow, that divide the bilayer into segments. (d) The length of the segments is reduced when the number of fiber doubles, resulting in smoother bending curvature. (e) When the fiber is oriented at $60^{\circ}$, the bending axis of the beam changed, forming a cylinder with the same orientation as the fiber. (f) The addition of more $60^{\circ}$-oriented fibers increase the bending stability but the overall shape of the bilayer remain the same. (g) However, the addition of fibers with radial orientation changed the bending shape to cone in which the tip originated from the center of the radial pattern. (h) Since the curvature of the bending change along the length of the fibers, adding more fiber helps formed the actuated bilayer into more distinct cone shape. Note: (b-d) and (e-f) are actuated with $5.5 \mathrm{kV}$ and $6.5 \mathrm{kV}$, respectively. 